\title{
On Monte Carlo Simulation for the HJM Model Based on Jump
}

\author{
Kisoeb Park ${ }^{1}$, Moonseong $\mathrm{Kim}^{2}$, and Seki Kim ${ }^{1, \star}$ \\ 1 Department of Mathematics, Sungkyunkwan University \\ 440-746, Suwon, Korea \\ Tel.: +82-31-290-7030, 7034 \\ $\{$ kisoeb, skim\}@skku.edu \\ 2 School of Information and Communication Engineering \\ Sungkyunkwan University \\ 440-746, Suwon, Korea \\ Tel.: +82-31-290-7226 \\ moonseong@ece.skku.ac.kr
}

\begin{abstract}
We derive a form of the HJM model based on jump. Heath, Jarrow, and Morton(HJM) model is widely accepted as the most general methodology for term structure of interest rate models. We represent the HJM model with jump and give the analytic proof for the HJM model with jump. We perform the Monte Carlo simulation with several scenarios to achieve highly precise estimates with the brute force method in terms of mean standard error which is one measure of the sharpness of the point estimates. We have shown that bond prices in HJM jump-diffusion version models of the extended Vasicek and CIR models obtained by Monte Carlo simulation correspond with the closed form values.
\end{abstract}

\section{Introduction}

Approaches to modeling the term structure of interest rates in continuous time may be broadly described in terms of either the equilibrium approach or the no-arbitrage approach even though some early models include concepts from both approaches. The no-arbitrage approach starts with assumptions about the stochastic evolution of one or more underlying factors, usually interest rate. Bond prices are assumed to be functions of the these driving stochastic processes.

Heath, Jarrow and Morton (HJM) 4 is widely accepted as the most general methodology for term structure of interest rate models. The major contribution of the HJM model [4, as it allows the model to be no-arbitrage, a major improvement over the Ho and Lee [5] and other similar models. We will represent the HJM model with jump. In pricing and hedging with financial derivatives, jump models are particularly important, since ignoring jumps in financial prices will cause pricing and hedging rates. Term structure model solutions under HJM model with jump is justified because movements in forward rates display both continuous and discontinuous behavior. These jumps are caused by several market

\footnotetext{
^ Corresponding author.
} 
phenomena money market interventions by the Fed, news surprise, and shocks in the foreign exchange markets, and so on. The HJM model with jump uses as the driving stochastic dynamic variable forward rates whose evolution is dependent on a specified volatility function. The most models of forward rates evolution in the HJM framework result in non-Markovian models of the short term interest rate evolution. This model depend on the entire history of forward rates. Therefore, this model is difficult of the actual proof analysis of the HJM model with jump. In this study, we got achieved to make the actual proof analysis of the HJM model with jump easy. The HJM model with volatility function was studied by Hull and White, Carverhill, Ritchken and Sankarasubramanian (RS) 9], Inui and Kijima, and Bhar and Chiarella in their attempt to obtain Markovian transformation of the HJM model. We examines the one-factor HJM model with jump which we use restrictive condition of RS.

We investigate the restrictive condition of $\mathrm{RS}$. In addition, we introduce the Monte Carlo simulation. One of the many uses of Monte Carlo simulation by financial engineers is to place a value on financial derivatives. Interest in use of Monte Carlo simulation for bond pricing is increasing because of the flexibility of the methods in handling complex financial institutions. One measure of the sharpness of the point estimate of the mean is Mean Standard Error. Numerical methods that are known as Monte Carlo methods can be loosely described as statistical simulation methods, where statistical simulation is defined in quite general terms to be any method that utilizes sequences of random numbers to perform the simulation.

The structure of the remainder of this paper is as follows. In the section 2 , the HJM model with jump are introduced. In the section 3, we calculate numerical solutions using Monte Carlo simulation for the HJM model with jump. In the section 4, we investigate the HJM model with the jump version of the extended Vasicek and CIR models. This paper is finally concluded in section 5 .

\section{Heath-Jarrow-Merton(HJM) Model with Jump}

The HJM consider forward rates rather than bond prices as their basic building blocks. Although their model is not explicitly derived in an equilibrium model, the HJM model is a model that explains the whole term structure dynamics in a no-arbitrage model in the spirit of Harrison and Kreps [6, and it is fully compatible with an equilibrium model. If there is one jump during the period $[t, t+d t]$ then $d \pi(t)=1$, and $d \pi(t)=0$ represents no jump during that period. We will ignore taxes and transaction costs. We denote by $V(r, r, T)$ the price at time $t$ of a discount bond. It follows immediately that $V(r, T, T)=1$. We consider the multi-factor HJM model with jump of term structure of interest rate is the stochastic differential equation(SDE) for forward rate

$$
d f(t, T)=\mu_{f}(t, T) d t+\sum_{i=1}^{n} \sigma_{f_{i}}(t, T) d W_{i}(t)+\sum_{i=1}^{n} J_{i} d \pi_{i}(t)
$$


where, $\mu_{f}(t, T)$ represents drift function; $\sigma^{2} f_{i}(t, T)$ is volatility coefficients; $J_{i}$ is the magnitude of a jump with $J_{i} \sim N\left(\theta, \delta^{2}\right)$; in this stochastic process n independent Wiener processes and Poisson processes determine the stochastic fluctuation of the entire forward rate curve starting from a fixed initial curve.

The main contribution of the HJM model is the parameters $\mu_{f}(t, T)$ and $\sigma_{f_{i}}(t, T)$ cannot be freely specified; drift of forward rates under the risk-neutral probability are entirely determined by their volatility and by the market price of risk. We introduce the no-arbitrage condition as follows:

$$
\mu_{f}(t, T)=-\sum_{i=1}^{n} \sigma_{f_{i}}(t, T)\left(\lambda_{i}(t)-\int_{t}^{T} \sigma_{f_{i}}(t, s) d s\right)
$$

where, $\lambda_{i}(t)$ represents the instantaneous market price of risk and that is independent of the maturity $T$. Furthermore, by an application of Girsanov's theorem the dependence on the market price of interest rate risk can be absorbed into an equivalent martingale measure. Thus, the Wiener processes is

$$
d W_{i}^{Q}(t)=d W_{i}(t)+\lambda_{i}(t) d s
$$

We consider the one-factor HJM model with jump of the term structure of interest rate(that is, $n=1$ ). Substituting the above the equation into no-arbitrage condition(3), we represent the stochastic integral equation the following:

$$
\begin{aligned}
f(t, T)-f(0, T) & =\int_{0}^{t} \sigma_{f}(u, T) \int_{t}^{T} \sigma_{f}(t, s) d s d u \\
& +\int_{0}^{t} \sigma_{f}(s, T) d W^{Q}(s)+\sum_{j=1}^{\pi(t)} J_{j}
\end{aligned}
$$

where, $d W_{i}^{Q}$ is the Wiener process generated by an equivalent martingale measure $Q$. The spot rate $r(t)=f(t, t)$ is obtained by setting $T=t$ in the equation (5), so that

$$
r(t)=f(0, t)+\int_{0}^{t} \mu_{f}(s, T) d s+\int_{0}^{t} \sigma_{f}(s, T) d W^{Q}(s)+\sum_{j=1}^{\pi(t)} J_{j}
$$

where, $\mu_{f}(t, T)=\sigma_{f}(t, T) \int_{t}^{T} \sigma_{f}(t, s) d s$, and $d W^{Q}(t)$ is a standard Wiener process generated by the risk-neutral measure $Q$. Under the corresponding riskneutral measure $Q$, the explicit dependence on the market price of risk can be suppressed, and we obtain the differential form of (3) is given by

$$
d f(t, T)=\mu_{f}(t, T) d t+\sigma_{f}(t, T) d W^{Q}(t)+J d \pi .
$$

We know that the zero coupon bond prices are contained in the forward rate informations, as bond prices can be written down by integrating over the forward rate between $t$ and $T$ in terms of the risk-neutral process

$$
V(t, T)=\exp \left(-\int_{t}^{T} f(t, s) d s\right) .
$$


From the equation (3), we derive the zero coupon bond prices as follow:

$$
\begin{aligned}
V(t, T) & =e^{-\int_{t}^{T} f(t, s) d s} \\
& =\frac{V(0, T)}{V(0, t)} e^{-\left(\int_{0}^{t} \int_{t}^{T} \mu_{f}(u, s) d s d u+\int_{0}^{t} \int_{t}^{T} \sigma_{f}(u, s) d s d W^{Q}(u)+\sum_{j=1}^{\pi(t)} J_{j} \int_{t}^{T} d s\right)}
\end{aligned}
$$

where, we define as $V(0, t)=e^{-\int_{0}^{t} f(0, s) d s}, V(0, T)=e^{-\int_{0}^{T} f(0, s) d s}$, and $\mu_{f}(t, T)=\sigma_{f}(t, T) \int_{t}^{T} \sigma_{f}(t, s) d s$.

The most models of forward rates evolution in the HJM framework result in non-Markovian models of the short term interest rate evolution. As above the equation (7), these integral terms depend on the entire history of the process up to time $t$. But, numerical methods for Markovian models are usually more efficient than those necessary for non-Markovian models.

We examines the one-factor HJM model with jump which we use restrictive condition of RS 9 . RS have extended Carverhill results showing that if the volatilities of forward rates were differential with respect to maturity date, for any given initial term structure, if and only if for the prices of all interest rate contingent claims to be completely determined by a two-state Markov process is that the volatility of forward rate is of the form

$$
\sigma_{f}(t, T)=\sigma_{r}(t) \exp \left(-\int_{t}^{T} a(s) d s\right)
$$

where, $\sigma_{r}$ and $a$ are deterministic functions. For the volatility of forward rate is of the form (8), the following formula for the discount bond price $V(t, T)$ was obtained in restrictive condition of RS.

Theorem 1. Let $\sigma_{f}(t, T)$ be as given in (8), then discount bond price $V(t, T)$ is given by the formula

$$
\begin{gathered}
\left.\qquad(t, T)=\frac{V(0, T)}{V(0, t)} \exp \left\{-\frac{1}{2} \varphi^{2}(t, T) \phi(t)+\varphi(t, T) \xi(t)\right]\right\} \\
\text { where, }\left\{\begin{array}{l}
\varphi(t, T)=\int_{t}^{T} \exp \left(-\int_{u}^{t} a(s) d s\right) d u \\
\phi(t)=\int_{0}^{t} \sigma_{f}^{2}(s, t) d s \\
\xi(t)=[f(0, t)-r(t)]
\end{array}\right.
\end{gathered}
$$

As we mentioned already, a given model in the HJM model with jump will result in a particular behavior for the short term interest rate. We introduce relation between the short rate process and the forward rate process as follows. In this study, we jump-diffusion version of Hull and White model to reflect this restriction condition. We know the following model for the interest rate $r$;

$$
d r(t)=a(t)[\theta(t) / a(t)-r(t)] d t+\sigma_{r}(t) r(t)^{\beta} d W^{Q}(t)+J d \pi(t),
$$

where, $\theta(t)$ is a time-dependent drift; $\sigma_{r}(t)$ is the volatility factor; $a(t)$ is the reversion rate. We will investigate the $\beta=0$ case is an extension of Vasicek's jump diffusion model; the $\beta=0.5$ case is an extension of CIR jump diffusion model. 
Theorem 2. Let be the jump-diffusion process in short rate $r(t)$ is the equation (10). Let be the volatility form is

$$
\sigma_{f}(t, T)=\sigma_{r}(t)(\sqrt{r(t)})^{\beta} \eta(t, T)
$$

with $\eta(t, T)=\exp \left(-\int_{t}^{T} a(s) d s\right)$ is deterministic functions. We know the jumpdiffusion process in short rate model and the "corresponding" compatible HJM model with jump

$$
d f(t, T)=\mu_{f}(t, T) d t+\sigma_{f}(t, T) d W^{Q}(t)+J d \pi(t)
$$

where $\mu_{f}(t, T)=\sigma_{f}(t, T) \int_{t}^{T} \sigma_{f}(t, s) d s$. Then we obtain the equivalent model is

$$
\begin{aligned}
f(0, T) & =r(0) \eta(0, T)+\int_{0}^{T} \theta(t) \eta(s, T) d s \\
& -\int_{0}^{T} \sigma_{r}^{2}(s)\left(r(s)^{2}\right)^{\beta} \eta(s, T) \int_{s}^{T}(\eta(s, u) d u) d s
\end{aligned}
$$

that is, all forward rates are normally distributed. Note that we know that $\beta=0$ case is an extension of Vasicek's jump diffusion model; the $\beta=0.5$ case is an extension of CIR jump diffusion model.

Note that the forward rates are normally distributed, which means that the bond prices are log-normally distributed. Both the short term rate and the forward rates can become negative. As above, we obtain the bond price from the theorem 1. By the theorem 2, we drive the relation between the short rate and forward rate.

Corollary 1. Let be the HJM model with jump of the term structure of interest rate is the stochastic differential equation for forward rate $f(t, T)$ is given by

$$
d f(t, T)=\sigma_{f}(t, T) \int_{t}^{T} \sigma_{f}(t, s) d s d t+\sigma_{f}(t, T) d W^{Q}(t)+J d \pi(t)
$$

where, $d W_{i}^{Q}$ is the Wiener process generated by an equivalent martingale measure $Q$ and $\sigma_{f}(t, T)=\sigma_{r}(t)(\sqrt{r(t)})^{\beta} \exp \left(-\int_{t}^{T} a(s) d s\right)$.

Then the discount bond price $V(t, T)$ for the forward rate is given by the formula

$$
\begin{aligned}
V(t, T) & =\frac{V(0, T)}{V(0, t)} \exp \left\{-\frac{1}{2}\left(\frac{\int_{t}^{T} \sigma_{f}(t, s) d s}{\sigma_{f}(t, T)}\right)^{2} \int_{0}^{t} \sigma_{f}^{2}(s, t) d s\right. \\
& \left.-\frac{\int_{t}^{T} \sigma_{f}(t, s) d s}{\sigma_{f}(t, T)}[f(0, t)-r(t)]\right\}
\end{aligned}
$$

with the equation (13).

Note that we know that $\beta=0$ case is an extension of Vasicek's jump diffusion model; the $\beta=0.5$ case is an extension of CIR jump diffusion model. 


\section{Monte Carlo Simulation of the HJM Model with Jump}

Recent methods of bond pricing do not necessarily exploit partial differential equations(PDEs) implied by risk-neutral portfolios. They rest on converting prices of such assets into martingales. This is done through transforming the underlying probability distribution using the tools provided by the Girsanov's theorem. A risk-neutral measure is any probability measure, equivalent to the market measure $P$, which makes all discounted bond prices martingales.

We now move on to discuss Monte Carlo simulation. A Monte Carlo simulation of a stochastic process is a procedure for sampling random outcomes for the process. This uses the risk-neutral valuation result. The bond price can be expressed as:

$$
V(t, T)=E_{t}^{Q}\left[e^{-\int_{t}^{T} f(t, s) d s}\right]
$$

where, $E_{t}^{Q}$ is the expectations operator with respect to the equivalent riskneutral measure. Under the equivalent risk-neutral measure, the local expectation hypothesis holds(that is, $E_{t}^{Q}\left[\frac{d V}{V}\right]$ ). According to the local expectation hypothesis, the term structure is driven by the investor's expectations on future short rates. To execute the Monte Carlo simulation, we discretized the equation (15). We divide the time interval $[t, T]$ into $m$ equal time steps of length $\Delta t$ each(that is, $\left.\Delta t=\frac{T-t}{m}\right)$. For small time steps, we are entitled to use the discretized version of the risk-adjusted stochastic differential equation (14):

$$
f_{j}=f_{j-1}+\left[\sigma_{f}(t, T) \int_{t}^{T} \sigma_{f}(t, s) d s d t\right] \Delta t+\sigma_{f}(t, T) \varepsilon_{j} \sqrt{\Delta t}+J_{j} N_{\Delta t}
$$

where, $\sigma_{f}(t, T)=\sigma_{r}(t)(\sqrt{r(t)})^{\beta} \exp \left(-\int_{t}^{T} a(s) d s\right), j=1,2, \cdots, m, \varepsilon_{j}$ is standard normal variable with $\varepsilon_{j} \sim N(0,1)$, and $N_{\Delta t}$ is a Poisson random variable with parameter $h \Delta t$. Note that we know that $\beta=0$ case is an extension of Vasicek's jump diffusion model; the $\beta=0.5$ case is an extension of CIR jump diffusion model. We can investigate the value of the bond by sampling $n$ spot rate paths under the discrete process approximation of the risk-adjusted processes of the equation (16). The bond price estimate is given by:

$$
V(t, T)=\frac{1}{n} \sum_{i=1}^{n} \exp \left(-\sum_{j=0}^{m-1} f_{i j} \Delta t\right)
$$

where $f_{i j}$ is the value of the forward rate under the discrete risk-adjusted process within sample path $i$ at time $t+\Delta t$. Numerical methods that are known as Monte Carlo methods can be loosely described as statistical simulation methods, where statistical simulation is defined in quite general terms to be any method that utilizes sequences of random numbers to perform the simulation. The Monte Carlo simulation is clearly less efficient computationally than the numerical method. 
The precision of the mean as a point estimate is often defined as the half-width of a $95 \%$ confidence interval, which is calculated as

$$
\text { Precision }=1.96 \times M S E .
$$

where, $M S E=\nu / \sqrt{n}$ and $\nu^{2}$ is the estimate of the variance of bond prices as obtained from $n$ sample paths of the short rate:

$$
\nu^{2}=\frac{\sum_{i=1}^{n}\left[\exp \left(-\sum_{j=0}^{m-1} f_{i j} \Delta t\right)-\nu\right]}{n-1} .
$$

Lower values of Precision in Equation(18) correspond to sharper estimates. Increasing the number of $n$ is a brute force method of obtaining sharper estimates. This reduces the MSE by increasing the value of $n$. However, highly precise estimates with the brute force method can take a long time to achieve. For the purpose of simulation, we conduct three runs of 1,000 trials each and divide the year into 365 time steps.

\section{Experiments}

In this section, we investigate the HJM model with the jump version of the extended Vasicek and CIR models. In experiment 1, the parameter values are assumed to be $r=0.05, a=0.5, \theta=0.025, \sigma_{r}=0.08, \lambda=-0.5, t=0.05$, $\beta=0$, and $T=20$.

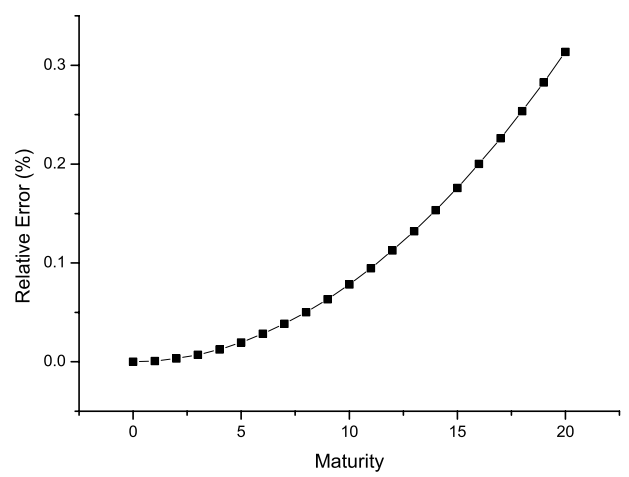

Fig. 1. Experiment 1: The relative error between the HJM model with the jump version of the extended Vasicek and CIR models

Experiment 2, contrasts bond prices by Monte Carlo simulation. In experiment 2 , the parameter values are assumed to be $r[0]=0.05, f[0, t]=0.049875878$, $a=0.5, \theta=0.025, \sigma_{r}=0.08, \lambda=-0.5, \beta=0, \Delta t=(T-t) / m, m=365$, $n=1000, t=0.05$, and $T=20$. 
Table 1. Experiment 2: Bond price estimated by the Monte Carlo simulation for the HJM model with the extended Vasicek model, CIR model, the jump diffusion version of the extended Vasicek model and CIR model.

\begin{tabular}{|c|c|c|c|c|}
\hline & HJME_V & HJME_CIR & Jump-HJME_V & Jump-HJME_CIR \\
\hline \hline CFS & 0.954902 & 0.95491 & 0.954902 & 0.95491 \\
\hline MCS & 0.951451 & 0.951456 & 0.951722 & 0.950465 \\
\hline CFS-MCS & $5.03495 \mathrm{E}-06$ & $1.27659 \mathrm{E}-06$ & 0.000319048 & 0.000289694 \\
\hline Variance & $7.09574 \mathrm{E}-05$ & 0.000112986 & 0.00178619 & 0.00170204 \\
\hline Precision & 0.00676342 & 0.00676933 & 0.00623192 & 0.00871051 \\
\hline
\end{tabular}

\section{Conclusion}

In this paper, we derive and perform the evaluation of the bond prices of the HJM-Extended Vasicek and the HJM-CIR models with forward interest rates instead of short rates using numerical methods. The results show that the values obtained are very similar. Even though it is hard to achieve the value of bond prices to term structure models when forward rates follow jump diffusions, we have shown that bond prices in HJM jump-diffusion version models of the extended Vasicek and CIR models obtained by Monte Carlo simulation correspond with the closed form solution. Lower values of precision in the HJM model with jump of the extended Vasicek model correspond to sharper estimates.

\section{References}

1. C. Ahn and H. Thompson, "Jump-Diffusion Processes and the Term Structure of Interest Rates," Journal of Finance, vol. 43, pp. 155-174, 1998

2. J. Baz and S. R. Das, "Analytical Approximations of the Term Structure for JumpDiffusion Processes: A Numerical Analysis," Journal of Fixed Income, vol. 6(1), pp. 78-86, 1996.

3. J. C. Cox, J. Ingersoll, and S. Ross, "A Theory of the Term Structure of Interest Rate," Econometrica, vol. 53, pp. 385-407, 1985.

4. D. Health, R. Jarrow, and A. Morton, "Bond Pricing and the Term Structure of Interest Rates," Econometrica, vol. 60, no.1, pp. 77-105, 1992.

5. T. S. Ho and S. Lee, "Term Structure Movements and Pricing Interest Rate Contingent Claims," Journal of Finance, vol. 41, pp. 1011-1028, 1986.

6. M. J. Harrison and D. M. Kreps, "Martingales and arbitrage in multiperiod securities markets," Journal of Economic Theory, vol. 20. pp. 381-408, 1979.

7. J. Hull and A. White, "Pricing Interest Rate Derivative Securities," Review of Financial Studies, vol. 3, pp. 573-92, 1990.

8. M. J. Brennan and E. S. Schwartz, "A Continuous Time Approach to the Pricing of Bonds," Journal of Banking and Finance, vol. 3, pp. 133-155, 1979.

9. P. Ritchken and L. Sankarasubramanian, "Volatility Structures of Forward Rates and the Dynamics of the Term Structure," Mathematical Finance, vol. 5, pp. 55-72, 1995.

10. O. A. Vasicek, "An Equilibrium Characterization of the Term Structure," Journal of Financial Economics, vol. 5, pp. 177-88, 1977. 